\title{
Compressibility in a Variable Generalised Chaplygin Gas
}

\author{
Manuel Malaver ${ }^{1,2}$ \\ ${ }^{1}$ Bijective Physics Institute, Idrija, Slovenia, and ${ }^{2}$ Maritime University of the Caribbean, Department of Basic Sciences, Catia la \\ Mar, Venezuela. \\ ${ }^{*}$ Corresponding author: Email: mmf.umc@gmail.com
}

(Received 22 January 2020; Accepted 14 May 2020)

\begin{abstract}
Considering the Panigrahi and Chatterjee model (2017) for variable generalised Chaplygin gas, in this paper we found for this kind of exotic matter an analytic expression for the adiabatic compressibility $\beta_{s}$. It was analyzed the behaviour of the adiabatic compressibility in the limit of high and low pressure. The derived equation for $\beta_{s}$ was used to deduce the value of the heat capacity at constant pressure $C_{p}$ for variable generalised Chaplygin gas.
\end{abstract}

Keywords: Adiabatic compressibility; Variable generalised Chaplygin Gas; Heat capacity; Exotic matter.

\section{Introduction}

The discovery of accelerating expansion of the universe (Amanullah et al., 2010; Kamenschick et al., 2001; Reiss et al., 1998) has allowed the opening new horizons in the field of physics and cosmology and the exotic matter model known as Chaplygin gas is one of the most explanations for this phenomena. Astronomical evidence (Spergel et al., 2007) has shown that the matter that makes up stars and galaxies is less than $5 \%$ of the universe's total mass and that much of the universe's total energy is in the form of dark energy and the rest as non-baryonic cold dark matter particles that has never been detected. The variable Chaplyging gas model was studied for Panigrahi (2015) and Malaver (2016). Panigrahi (2015) demonstrated that this model satisfies the third law of thermodynamics and Malaver (2016) derived an expression for the adiabatic compresibility in a variable Chaplygin gas model. More recently, Panigrahi and Chatterjee (2017) propose a variable generalized Chaplygin gas model and deduce some thermodynamic equations in terms of temperature and volume.

\section{Objective}

In this paper an expression has been deduced for the adiabatic compressibility of the variable generalised Chaplygin gas (VGCG) from the equation of state given for Panigrahi and Chatterjee (2017). With the equation obtained for the adiabatic compressibility we derived an expression for the heat capacity at constant pressure $C_{P}$ in a VGCG model. We found that the adiabatic compressibility for this model only will depend on the pressure and $C_{P}$ is always positive.

\section{Compressibility in a Variable Generalised Chaplygin Gas}

For a variable generalised Chaplygin gas (Panigrahi \& Chatterjee, 2017) the equation of state for the pressure is

\footnotetext{
(c) The Author(s), 2020. Published by Cambridge University Press. This is an Open Access article, distributed under the terms of the Creative Commons Attribution licence (http://creativecommons.org/licenses/by/4.0/), which permits unrestricted re-use, distribution, and reproduction in any medium, provided the original work is properly cited.
} 


$$
P=-\left(B_{0} V^{-\frac{n}{3}}\right)^{\frac{1}{1+\alpha}}\left(\frac{N}{1+\alpha}\right)^{\frac{\alpha}{1+\alpha}}\left(1-\left(\frac{T}{\tau}\right)^{\frac{1+\alpha}{\alpha}}\right)^{\frac{\alpha}{1+\alpha}}
$$

where $B_{0}$ is a positive universal constant, $N=\frac{3(1+\alpha)-n}{3}, n$ is a constant, $\tau$ is a universal constant with dimension of temperature and $\alpha$ is a parameter.

Following Zemansky and Dittman (1985), the adiabatic compressibility can be written as

$$
\beta_{S}=-\frac{1}{V}\left(\frac{\partial V}{\partial P}\right)_{S}=-\frac{1}{V}\left(\frac{\partial V}{\partial T}\right)_{S}\left(\frac{\partial T}{\partial P}\right)_{S}=-\frac{(\partial V / \partial T)_{S}}{V(\partial P / \partial T)_{S}}
$$

From the equation proposed by Malaver (2015) for a VGCG model

$$
V\left[1-\left(\frac{\tau}{T}\right)^{\frac{1+\alpha}{\alpha}}\right]^{-\frac{1}{N}}=\text { const. }
$$

We obtain

$$
\left(\frac{\partial V}{\partial T}\right)_{S}=\frac{\text { const. }}{N}\left[1-\left(\frac{\tau}{T}\right)^{\frac{1+\alpha}{\alpha}}\right]^{\frac{1-N}{N}}\left(\frac{1+\alpha}{\alpha}\right)\left(\frac{\tau^{2}}{T^{2+\alpha}}\right)^{\frac{1}{\alpha}}
$$

Substituting (3) in (4) we have

$$
\left(\frac{\partial V}{\partial T}\right)_{S}=\left(\frac{1+\alpha}{\alpha}\right) \frac{V}{N\left[1-\left(\frac{\tau}{T}\right)^{\frac{1+\alpha}{\alpha}}\right]}\left(\frac{\tau^{2}}{T^{2+\alpha}}\right)^{\frac{1}{\alpha}}
$$

According with Malaver (2017), the expression for an adiabatic reversible process for the VGCG model in terms of $P$ and $T$ is given by

$$
\frac{P T^{\frac{N-(1+\alpha)}{N}}}{\left[\left(\frac{T}{\tau}\right)^{\frac{1+\alpha}{\alpha}}-1\right]^{\frac{N-1}{N}}}=\text { const. }
$$

With the eq. (6), $\left(\frac{\partial P}{\partial T}\right)_{S}$ takes the form

$$
\left(\frac{\partial P}{\partial T}\right)_{S}=\text { const. }\left[\left(\frac{T}{\tau}\right)^{\frac{1+\alpha}{\alpha}}-1\right]^{-\frac{1}{N}} T^{\frac{1+\alpha-N}{N}}\left\{\left(\frac{\alpha+1}{\alpha}\right)\left(\frac{N-1}{N}\right)\left(\frac{T}{\tau^{1+\alpha}}\right)^{\frac{1}{\alpha}}-\left[\frac{N-(1+\alpha)}{N}\right]\left[\left(\frac{T}{\tau}\right)^{\frac{1+\alpha}{\alpha}}-1\right] \frac{1}{T}\right\}
$$

Replacing (6) in (7) and rearranging terms

$$
\left(\frac{\partial P}{\partial T}\right)_{S}=\frac{P\left\{\left(\frac{1+\alpha}{\alpha}\right)\left(\frac{N-1}{N}\right)\left(\frac{T}{\tau}\right)^{\frac{1+\alpha}{\alpha}}+\left[\frac{N-(1+\alpha)}{N}\right]\left[1-\left(\frac{T}{\tau}\right)^{\frac{1+\alpha}{\alpha}}\right]\right\}}{T\left[\left(\frac{T}{\tau}\right)^{\frac{1+\alpha}{\alpha}}-1\right]}
$$

Substituting (5) and (8) in eq. (2) we have 


$$
\beta_{S}=\left(\frac{1+\alpha}{\alpha P}\right)\left(\frac{\tau}{T}\right)^{\frac{2}{\alpha}} \frac{\left[1-\left(\frac{T}{\tau}\right)^{\frac{1+\alpha}{\alpha}}\right]}{\left[1-\left(\frac{\tau}{T}\right)^{\frac{1+\alpha}{\alpha}}\right]\left\{(1+\alpha)(N-1)\left(\frac{T}{\tau}\right)^{\frac{1+\alpha}{\alpha}}+[N-(1+\alpha)]\left[1-\left(\frac{T}{\tau}\right)^{\frac{1+\alpha}{\alpha}}\right]\right\}}
$$

The expression for the adiabatic compressibility in the VGCG model (9) is an explicit function of the temperature and the pressure and can be used to calculate the heat capacity at constant pressure from the following equation (Zemansky \& Dittman,1985)

$$
\frac{C_{P}}{C_{V}}=\frac{\beta_{T}}{\beta_{S}}
$$

The isothermal compressibility $\beta_{T}$ can be written as

$$
\beta_{T}=-\frac{(1+\alpha)}{[N-(1+\alpha)] P}
$$

With the equations (9) and (11) we obtain

$$
\frac{\beta_{T}}{\beta_{S}}=\left(\frac{T}{\tau}\right)^{\frac{2}{\alpha}\left(\left(\frac{\tau}{T}\right)^{\frac{1+\alpha}{\alpha}}-1\right)\left\{(1+\alpha)(N-1)\left(\frac{T}{\tau}\right)^{\frac{1+\alpha}{\alpha}}+[N-(1+\alpha)]\left[1-\left(\frac{T}{\tau}\right)^{\frac{1+\alpha}{\alpha}}\right]\right\}}
$$

The heat capacity at constant volume for a variable generalised Chaplygin gas (Panigrahi \& Chatterjee,2017) is given by

$$
C_{V}=\frac{\left[\frac{B_{0}(1+\alpha) V^{N}}{N}\right]^{\frac{1}{1+\alpha}}\left(\frac{T}{\tau^{1+\alpha}}\right)^{\frac{1}{\alpha}}}{\alpha\left\{1-\left(\frac{T}{\tau}\right)^{\frac{1+\alpha}{\alpha}}\right\}^{\frac{2+\alpha}{1+\alpha}}}
$$

And from the eq. (12) and eq. (13), $C_{P}$ can be written as

$$
C_{P}=\left[\frac{B_{0}(1+\alpha)}{N}\right]^{\frac{1}{1+\alpha}}\left(\frac{T^{3}}{\tau^{3+\alpha}}\right)^{\frac{1}{\alpha}} V^{\frac{N}{1+\alpha}} \frac{\left[\left(\frac{\tau}{T}\right)^{\frac{1+\alpha}{\alpha}}-1\right]\left\{(1+\alpha)(N-1)\left(\frac{T}{\tau}\right)^{\frac{1+\alpha}{\alpha}}+[N-(1+\alpha)]\left[1-\left(\frac{T}{\tau}\right)^{\frac{1+\alpha}{\alpha}}\right]\right\}}{\alpha[N-(1+\alpha)]\left[1-\left(\frac{T}{\tau}\right)^{\frac{1+\alpha}{\alpha}}\right]^{\frac{3+2 \alpha}{1+\alpha}}}
$$

$C_{P}>0$, this implies that $n<0, \alpha>0$ and $\tau>0$. From the thermodynamic stability considerations $n$ must have a negative value (Panigrahi, 2015). With $\alpha=1$ is recovered the expression for $C_{P}$ of variable Chaplygin gas obtained by Malaver (2016) as a particular case of this work.

\section{Conclusions}

We obtained an expression for the adiabatic compressibility of a variable generalised Chaplygin gas in terms of the pressure, temperature and $\alpha$ parameter. Is predicted that for $\beta_{S} \rightarrow \infty$ when $P \rightarrow 0$ and $\beta_{s} \rightarrow 0$ if $P \rightarrow \infty$ as in the ideal gas. Furthermore, with the equation for $\beta_{S}$ we found a new equation for the heat capacity at constant pressure $C_{P}$ for VGCG model that depends only the temperature and parameter $\alpha$ and that always is positive for $n<0, \alpha>0$ and $0<T<\tau$.

Funding Information. This research received no specific grant from any funding agency, commercial or not-for-profit sectors. 
Conflict of interest. The author declare that there is no conflict of interest regarding the publication of this article.

Data Availability. The data that support the findings of this study are openly available in https://arxiv.org/abs/1608.00244, Gen.Rel.Grav. 49 (2017), no.3, 35.

\section{References}

Amanullah, R., Lidman, C., Rubin, D., Aldering, G., Astier, P., Barbary, K., Burns, M. S., Conley, A., Dawson, K. S., Deustua, S. E., Doi, M., Fabbro, S., Faccioli, L., Fakhouri, H. K., Folatelli, G., Fruchter, A. S., Furusawa, H., Garavini, G., Goldhaber, G., ... Hook, I. (2010). Spectra and light curves of six type Ia supernovae at $0.511<z<1.12$ and the Union2 compilation. Astrophysical Journal, 716, 712-738.

Kamenschick, A., Moschella, U., \& Pasquier, V. (2001). An alternative to quintessence. Physics Letters, B511, $265-268$.

Malaver, M. (2015). Carnot engine model in a Chaplygin gas. Research Journal of Modeling and Simulation, 2, 42-47.

Malaver, M. (2016). Adiabatic compressibility of the variable Chaplygin gas. AASCIT Communications, 3, 64-70.

Malaver, M. (2017). Thermodynamical analysis for a variable generalized Chaplygin gas. World Scientific News, 66, $149-162$.

Panigrahi, D. (2015). Thermodynamical behaviour of the variable Chaplygin gas. International Journal of Modern Physics, D24, 1550030. doi:https://doi.org/10.1142/S0218271815500303.

Panigrahi, D., \& Chatterjee, S. (2017). Viability of variable generalised Chaplygin gas-A thermodynamical approach. GenRelGrav, 49, 35.

Reiss, A. G., Filippenko, A. V., Challis, P., Clocchiattia, A., Diercks, A., Garnavich, P. M., Gilliland, R. L., Hogan, C. J., Jha, S., Kirshner, R. P., Leibundgut, B., Phillips, M. M., Reiss, D., Schmidt, B. P., Schommer, R. A., Smith, R. C., Spyromilio, J., Stubbs, C., Suntzeff, N. B., ... Tonry, J. (1998). Observational evidence from supernovae for an accelerating universe and a cosmological constant. Astronomical Journal, 116, 1009-1038.

Spergel, D. N., Bean, R., Doré, O., Nolta, M. R., Bennett, C. L., Dunkley, J., Hinshaw, G., Jarosik, N., Komatsu, E., Page, L., Peiris, H. V., Verde, L., Halpern, M., Hill, R. S., Kogut, A., Limon, M., Meyer, S. S., Odegard, N., Tucker, G. S., ... Wright, E. L. (2007). Wilkinson Microwave Anisotropy Probe (WMAP) three year results: Implications for cosmology. Astrophysical Journal Supplement, 170, 377.

Zemansky, M. W., \& Dittman, R. H. (1985). McGraw-Hill Interamericana. ISBN: 968-451-631-2.

Cite this article: Malaver M (2020). Compressibility in a Variable Generalised Chaplygin Gas Experimental Results, 1, e25, 1-8. https://doi.org/10.1017/exp.2020.21 


\section{Peer Reviews}

Reviewing editor: Prof. Stefano Camera ${ }^{1,2}$

${ }^{1}$ Universita degli Studi di Torino, Physics, Via Pietro Giuria, 1, Torino, Italy, 10124

${ }^{2}$ University of the Western Cape, Physics \& Astronomy, Bellville, South Africa, 7535

This article has been accepted because it is deemed to be scientifically sound, has the correct controls, has appropriate methodology and is statistically valid, and met required revisions.

doi:10.1017/exp.2020.21.pr1

\section{Review 1: Compressibility in a Variable Generalised Chaplygin Gas}

Reviewer: Hamed Kasmaei (D)

Islamic Azad University Central Tehran Branch, Department of Applied Mathematics

Date of review: 06 February 2020

Published online:

(C) The Author(s) 2020 This is an Open Access article, distributed under the terms of the Creative Commons Attribution licence (http://creativecommons.org/licenses/by/4.0/), which permits unrestricted re-use, distribution, and reproduction in any medium, provided the original work is properly cited.

Conflict of interest statement. Reviewer declares none

Comments to the Author: This paper has been written very well. My vote to this paper is minor revision. I will accept the paper after doing modifications. The reason is: The paper does not include results and discussion part. Also, it needs to be investigated that there is needed to provide some graphs and tables to show results in tabular and illustrative format. Also, it needs to be explained more about applications of Generalized Chaplygin Gas model in this paper and also its difference with its classic format to analyze results. I hope these comments help the author about improvement of this paper.

\section{Score Card}

Presentation

5.0

Is the article written in clear and proper English? (30\%)

Is the data presented in the most useful manner? (40\%)

Does the paper cite relevant and related articles appropriately? (30\%)

Context

Does the title suitably represent the article? (25\%)

Does the abstract correctly embody the content of the article? (25\%)

Does the introduction give appropriate context? (25\%)

Is the objective of the experiment clearly defined? (25\%) 
Are the limitations of the experiment as well as the contributions of the experiment clearly outlined? (20\%) 


\title{
Review 2: Compressibility in a Variable Generalised Chaplygin Gas
}

\author{
Reviewer: Dr. Amrit Sorli iD \\ Bijective Physics Institute, Gorenja Trebuša 79, Idrija, Slovenia, 5280 \\ Date of review: 26 March 2020 \\ Published online:
}

\begin{abstract}
(c) The Author(s) 2020 This is an Open Access article, distributed under the terms of the Creative Commons Attribution licence (http://creativecommons.org/licenses/by/4.0/), which permits unrestricted re-use, distribution, and reproduction in any medium, provided the original work is properly cited.
\end{abstract}

Conflict of interest statement. Reviewer declares none

Comments to the Author: Cosmology progress is based on experimental data not on theoretical speculations. "Chaplygin gas" is a theoretical exotic idea, it is not experimental data. Experimental data is that universal space has Euclidean shape which is measured by NASA in 2014. This means the volume of the universal space is infinite. Results of Barbour, Fiscaletti, Sorli confirm that time has no physical existence, time is the numerical sequential order of events running in the universal space, which is the primordial energy of the universe we call today "superfluid quantum vacuum", see work of Sbitnev, Fedi, Fiscaletti, Sorli. The energy of the vacuum itself is $95 \%$ of the missing energy of the universe, see the article published in Scientific Reports in August 2019: "Mass-energy Equivalence Extention on Superfluid Quantum Vacum". Universal space is time-invariant (as time is the numerical sequential order of change in space). Cosmological principle is time-invariant. The idea of the beginning of the universe from some singularity as proposed by Hawking and Hartle seems is not the best idea because nobody knows how a mathematical point can turn into infinite Euclidean space. Big Bang cosmology calculated the age of the universe is controversial with a measured diameter of the observable universe. According to BB cosmology universe to reach today size should expand with 3,34 of light speed. This does not make sense and I think this article somehow could be accepted 20 years ago. But not today. According to the measured observed universe, $\mathrm{CMB}$ could not reach us yet. See the recent article A THREEDIMENSIONAL NON-LOCAL QUANTUM VACUUM AS THE ORIGIN OF PHOTONS in the Ukrainian Journal of physics (Fiscaletti, Sorli). CMB is the radiation of the existent quantum vacuum. And the gravitational redshift of the light coming from distant galaxies can be seen as a "tired light effect" proposed by Zwicky. Direct reading of data is not in favor of BB cosmology.

\section{Score Card}

Presentation

Is the article written in clear and proper English? (30\%)

Is the data presented in the most useful manner? (40\%)

Does the paper cite relevant and related articles appropriately? (30\%) 
Does the introduction give appropriate context? (25\%)

Is the objective of the experiment clearly defined? (25\%)

Analysis

Does the discussion adequately interpret the results presented? (40\%)

Is the conclusion consistent with the results and discussion? (40\%)

Are the limitations of the experiment as well as the contributions of the experiment clearly outlined? (20\%) 